\title{
ProMACE-CytaBOM Regimen
}

National Cancer Institute

\section{Source}

National Cancer Institute. ProMACE-CytaBOM Regimen. NCI Thesaurus. Code C63460.

A non-cross resistant regimen consisting of prednisone, methotrexate, doxorubicin,

cyclophosphamide and etoposide (ProMACE) alternating with cytarabine, bleomycin, vincristine and methotrexate (CytaBOM), used to treat aggressive forms of non-Hodgkin lymphoma. 